\title{
Adaptive Cytoprotection and the Brain-Gut Axis
}

\author{
J.K.S. Ko ${ }^{\text {a }}$ C.H. Cho ${ }^{b}$ \\ ${ }^{a}$ Center for Cancer and Inflammation Research, School of Chinese Medicine, Hong Kong Baptist University, and \\ ${ }^{b}$ School of Biomedical Sciences, Faculty of Medicine, The Chinese University of Hong Kong, Hong Kong, SAR, China
}

\section{Key Words}

Adaptive cytoprotection - Mild irritants - Gastric mucosa • Prostaglandins - Vagus nerve $\cdot$ Afferent sensory neurons $\cdot$ Hypothalamic-pituitary-adrenal axis

\begin{abstract}
Adaptive cytoprotection is a concept to counteract against the gastric mucosal injury caused by stress, strong irritants and drugs such as non-steroidal anti-inflammatory drugs. The process is mediated through diverse mediators and mechanisms. Studies on adaptive cytoprotection began from the discovery of prostaglandin (PG)-dependent and PG-independent pathways, followed by the investigation on the types and concentrations of mild irritants to be used. Upon the confirmation on the importance of the vagus nerve and the vago-vagal pathway in regulating the mucosal protective actions of the mild irritants, individual participating mediators for the neuronal modulatory processes were explored, including peptide neurotransmitters such as calcitonin gene-related peptide and substance P. Further correlation with the sympathetic nervous system, the sensory afferent neurons and the enteric nervous system of the gastric mucosa had been made. A close working relationship between the hypothalamic-pituitary-adrenal axis, the autonomic nervous system and the enteric nervous system was
\end{abstract}

then proposed, with concurrent regulation of PG, nitric oxide and sensory neuropeptides by different mild irritants. Apart from these conventional concepts, there are now contemporary ideas on newer forms of adaptive cytoprotection such as ischemic preconditioning and heat-shock proteins, which will cast new light to novel approaches in facilitating gastric mucosal protection.

Copyright $\odot 2011$ S. Karger AG, Basel

\section{Cytoprotection and Adaptive Cytoprotection}

Gastric cytoprotection had been classically defined as the property of certain substances, particularly prostaglandins (PG), to protect the gastric mucosa from becoming inflamed and necrotic when exposed to noxious agents [1]. The protection occurs under the surface epithelium, which is susceptible to be injured upon oral administration of ethanol. With the pretreatment of PG, the deeper layers that constitute more than $90 \%$ of the thickness of the gastric mucosa can be protected. The use of the term 'cytoprotection' has been challenged by some investigators throughout the years due to its non-specificity. Szabo [2] had criticized the word 'cytoprotection' by showing that PG only protects against deep hemorrhagic necrosis in the mucosa without altering the initial

\section{KARGER}

Fax +4161306 1234

E-Mail karger@karger.ch

www.karger.com
(C) 2011 S. Karger AG, Basel

$0012-2823 / 11 / 0835-0019 \$ 38.00 / 0$

Accessible online at:

www.karger.com/dig
Dr. J.K.S. Ko

Center for Cancer and Inflammation Research

School of Chinese Medicine, Hong Kong Baptist University

Hong Kong, SAR (China) 
damage to surface epithelial cells. In general, three major types of cytoprotection have been classified, namely 'substitution', 'direct' and 'adaptive cytoprotection'. Gastric mucosal damage could be produced by agents that deplete PG content in biological tissues. This is the case when non-steroidal anti-inflammatory drugs inhibit the activity of the cyclooxygenase. Gastric lesions produced by these drugs (e.g. aspirin, indomethacin, etc.) are linked to gastric mucosal PG deficiency. Correction of this deficiency and prevention of mucosal damage could be achieved by PG replenishment, which is designated as 'substitution cytoprotection'. The second type of cytoprotection is related to protection against gastric lesions induced by agents other than non-steroidal anti-inflammatory drugs, such as strong ethanol, acids and bases. These agents destroy the mucosa and the underlying cells by direct damaging action. They cause lesions in the gastric mucosa that contains normal amounts of PG, of which PG supplementation is useful in the prevention of lesion formation. The third type of cytoprotection involves the endogenous stimulation of PG formation, above basal levels, by certain agents placed in the gastric lumen. This is the case of mild irritants which protect the stomach from damage caused by strong irritants [3]. Among the three types of cytoprotection, the implication of adaptive cytoprotection is more critical than others because it involves modulation of the normal defensive mechanism in the gastric mucosa, which potentially contributes to new drug development in treating ulcer diseases.

At the early stage of cytoprotection studies, investigators believed that there is a crucial involvement of PG in adaptive cytoprotection [4]. Nevertheless, others soon proposed that adaptive cytoprotection induced by low concentrations of ethanol, sodium chloride $(\mathrm{NaCl})$ and hydrochloric acid $(\mathrm{HCl})$ may not be necessarily mediated by prostanoids, at least under certain physiological conditions. It appears that at least some forms of adaptive cytoprotection do not require an intact cyclooxygenase system. Hence, the possibility of having other potential mediators of adaptive cytoprotection had been evolved after the discovery of certain protective substances in the gastric luminal fluid of rats receiving a low concentration of $\mathrm{HCl}$, which was unaffected by indomethacin. It was found that adaptive cytoprotection could be weakened or completely abolished after surgical sialoadenectomy, vagotomy or sympathectomy [5]. Since there is no unifying hypothesis regarding the mediation of adaptive cytoprotection, it is reasonable to assume that more than one mediator is involved in its mode of action, with the participation of multiple biological systems.

\section{Neuronal Mediators of Gastric Mucosal Defense}

It is known that neuronal modulatory processes and the release of local mediators are crucial for the gastric mucosa to resist the continual onslaught of aggressive agents. Release of vasodilator neuropeptides from afferent sensory neurons through the local reflex arc was suggested to be a protective mechanism in the gastric mucosa [6]. The stomach is densely innervated by primary sensory neurons containing peptide neurotransmitters, including calcitonin gene-related peptide (CGRP), substance $\mathrm{P}(\mathrm{SP})$ and tachykinins, and such neurons are found to be in close proximity to the submucosal microvasculature. CGRP could play the role as an endogenous vasoactive mediator involving in the regulation of gastric blood flow and mucosal integrity [7]. Besides, there is a close association of SP- and CGRP-containing neurons with mucosal mast cells [8], while local release of SP may play a role in inactivating CGRP. In other words, inhibition of SP release could increase the effectiveness of CGRP. Interaction between the endothelium-derived vasodilator mediators CGRP and prostacyclin had been reported in the regulation of gastric mucosal microcirculation and integrity [9]. Nonetheless, capsaicin administration attenuates the protective properties of $\mathrm{PGE}_{2}$ and its analogue against acute gastric challenge, again suggesting a permissive role for sensory neuropeptides in the mechanisms of protection by this prostanoid [10].

\section{Importance of the Vagus Nerve and the Enteric Nervous System in Modulating Ethanol-Induced Gastric Mucosal Damage and Adaptive Cytoprotection by Mild Irritants}

Electrical vagal stimulation had been shown to increase the incidence of hemorrhagic ulcers in the rat glandular mucosa, which substantiated the idea that stressinduced glandular ulcers could be resulted from vagal overactivation [11]. Vagotomy is able to decrease the injury caused by severe stress, acetylsalicylic acid and $\mathrm{HCl}$. On the other hand, damage to the mucosa by noxious agents such as strong ethanol would be worsened after truncal vagotomy, along with the abolition of the cytoprotective ability of 16,16 -dimethyl-PGE 2 [12]. Taken together, it appears that the autonomic nervous system (ANS) is important in the gastric defense against ethanolinduced mucosal injury. Vagotomy could result in aggravation of gastric mucosal lesion formation, despite its effectiveness in acid suppression [13]. Even in cases with 
successful healing, the high relapse rate as well as other unexpected severe side effects (including mortality) after vagotomy all suggest that it may not be recommended as a treatment of choice for gastric ulcers. Bilateral subdiaphragmatic truncal vagotomy results in small changes in mucosal architecture with varying degrees of functional changes, which could affect the integrity of the gastric mucosa. Electrical stimulation to one of the gastric branches of the vagus nerve could induce gastric lesions to the respective side of the mucosa, leading to the conclusion that the left and right gastric branches are innervated by the left and right dorsal motor nuclei of the vagus nerve in the medulla oblongata, respectively [14]. In fact, the vagus nerve contains a protective reflex arc as suggested by some investigators [6]. It however appears that the defensive actions of the functional side of the mucosa (with intact nerve) may not be able to protect against lesion formation on the denervated side.

Vagal denervation could weaken the mucus barrier and affect the protective action of acid inhibitors in the gastric mucosa. We had suggested that the vagal neuronal components could be involved in the adaptive cytoprotection of mild irritants through a vago-vagal reflex of the enteric nervous system. In our investigation of the possible intramucosal communication of adaptive cytoprotection using an ex-vivo chamber technique, it was found that the protective action of $20 \%$ ethanol and $0.3 \mathrm{M} \mathrm{HCl}$, but not of $5 \% \mathrm{NaCl}$, could be transmitted from one side to the contralateral side of the gastric mucosa that had not been preexposed to the mild irritant [15]. The differential effects of different mild irritants in protection transmission may be due to the higher infiltrating power of ethanol and $\mathrm{HCl}$ as compared to $\mathrm{NaCl}$, of which the former two compounds can enter the mucosa more easily and stimulate the transmission of the neuronal signal within the mucosal plexus. In addition, it was noted that only a small part of the cytoprotective effect of $20 \%$ ethanol, but the majority of the cytoprotective effect of $5 \% \mathrm{NaCl}$ on the protected side, still persisted in vagotomized animals when the protective reflex arc (the afferent sensory nerve) had been terminated. Nevertheless, the cytoprotection of $0.3 \mathrm{M} \mathrm{HCl}$ was completely blocked by vagotomy, indicating that an intact vagal tone is required in its action. This observation may be explained by the fact that following vagotomy, the physiological response of the oxyntic glands to the presence of $0.3 \mathrm{M} \mathrm{HCl}$ in the gastric lumen is lost, which in turn affects the adaptive cytoprotection by this mild irritant.

\section{Involvement of Different Components of the Autonomic Nervous System in the Adaptive Cytoprotection of Mild Irritants}

Co-localization of the neurotransmitter receptors and their binding sites for the cholinergic, adrenergic and dopaminergic nerve fibers was found throughout the stomach wall. Both ulcerogenic actions of ethanol and adaptive cytoprotection induced by mild irritants can be blocked by cholinergic receptor blockers [15]. In contrast, dopamine has shown a marked protective action during the pathogenesis of gastric lesion formation, which implies the involvement of the dopaminergic receptors in the mucosal defense mechanism [16]. The involvement of the sympathetic nervous system in the pathogenesis and cytoprotective action in the gastric mucosa remains controversial. We have shown that neither blockade of the

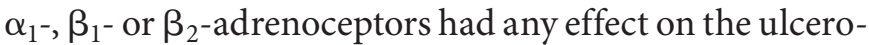
genic action of $100 \%$ ethanol, nor did they induce any alteration to the adaptive cytoprotection of mild irritants [17]. Nevertheless, $\alpha_{2}$-adrenoceptor antagonism significantly increased the severity of $100 \%$ ethanol-induced mucosal injury and completely blocked the cytoprotective action of $20 \%$ ethanol, although the effects of $5 \%$ $\mathrm{NaCl}$ and $0.3 \mathrm{M} \mathrm{HCl}$ were not affected. This result was supported by the report that $\alpha_{2}$-adrenoceptor agonists clonidine and $\alpha$-methyldopa possessed gastroprotective effects [18]. Blocking the $\alpha_{2}$-adrenoceptors would prevent the negative feedback action on the release of catecholamines from presynaptic nerve terminals, which could participate at least partly in the adaptive cytoprotection of $20 \%$ ethanol and the mucosal defensive mechanisms of the stomach in general. As a matter of fact, a similar modulation has also been achieved by blocking the dopamine $\mathrm{D}_{2}$-receptors, possibly be explained by the similar mechanism as in the case of $\alpha_{2}$-adrenoceptors blockade. These observations indicate that the autoregulatory mechanism of catecholamine release from adrenergic, and possibly dopaminergic nerve terminals could play a significant role in the defensive mechanism as well as the adaptive cytoprotection by mild irritants in the stomach.

\section{Implication of Sensory Afferent Neurons in the Mechanism of Gastric Adaptive Cytoprotection}

The vagus nerve is composed of only $10 \%$ efferent cholinergic and peptidergic fibers, whereas $90 \%$ are being afferent fibers. The latter component has demonstrated a crucial role in gastric mucosal defense since permanent 
denervation of the unmyelinated afferent neurons would induce gastric ulceration. Capsaicin-sensitive sensory fibers innervate the gastrointestinal tract of various animal species, and appear to play a relevant role in protecting the gastric mucosa from ulcerogenic agents. Cumulative doses of capsaicin injection were proven to completely destroy the unmyelinated sensory afferent fibers of the vagus nerve. Capsaicin-induced denervation of the sensory neurons abolished the laparotomy-induced protection against gastric mucosal injury caused by $75 \%$ ethanol [19]. This could explain the lesion-worsening effect of vagotomy, which terminates the capsaicin-sensitive sensory afferent fibers. Our previous findings indicate that capsaicin-induced desensitization of the sensory afferent neurons not only produced a more severe gastric mucosal damage after strong ethanol challenge, but also completely abolished the protective action of mild irritants such as $20 \%$ ethanol. A similar effect can be resulted by luminal instillation of lidocaine [17]. These observations suggest that adaptive cytoprotection induced by this mild irritant is at least partly mediated via the afferent sensory fibers. On the other hand, the cytoprotective effects of $5 \%$ $\mathrm{NaCl}$ and $0.3 \mathrm{M} \mathrm{HCl}$ were not affected by capsaicin, which means that their mode of action may not necessarily rely on this neuronal pathway.

\section{Hypothalamic-Pituitary-Adrenal Axis and Adaptive Cytoprotection in the Stomach}

In a schematic illustration of the brain-gut modulation over gastrointestinal functions, it has been demonstrated that activation of the hypothalamic-pituitary-adrenal (HPA) axis will lead to synthesis of corticosteroids and their release into the cardiovascular system [20]. Glucocorticoids released during acute activation of the HPA axis (e.g. mild stress) have been proven to be important gastroprotective factors, which contribute to adaptive cytoprotection [21]. A complementary pathway in turn involves activation of the ANS (sympathetic innervation) and results in the release of catecholamines into gastrointestinal tissues. Both HPA and ANS pathways are fundamental to the stress response as an outcome of injury and psychological stress. The influence on the cardiovascular system and the subsequent alteration of gastrointestinal functions is also simultaneously regulated by the enteric nervous system. Apart from these, the sensory nervous system plays a pivotal role in maintaining the integrity of the body against injury of any kind. The afferent function of sensory neurons serves to convey infor- mation about the type and site of injury to the central nervous system. Sensory neurons then transmit the efferent signal by releasing various sensory neuromodulators in the periphery. Another important function of sensory nerves is the protection of individual organs from being injury, including the stomach. It is now known that PG, nitric oxide (NO) as well as sensory neuropeptides including CGRP and SP may contribute to sustaining a favorable condition to counteract aggressive factors. It had been suspected that sensory neurons are not implicated in adaptive cytoprotection because no influence of capsaicin-sensitive neurons ablation on the gastroprotection of certain mild irritants $(0.2 \mathrm{M} \mathrm{HCl}$ and $20 \%$ ethanol) was observed under some experimental conditions. This speculation also points to the fact that adaptive cytoprotection may involve various mechanisms dependent on different types and concentrations of mild irritants [22]. For instance, sodium hydroxide and $\mathrm{HCl}$ do not require capsaicin-sensitive afferent neurons to provide NO- and PG-based adaptive cytoprotection. However, even with these observations, it should not be concluded that mild irritants do not stimulate the sensory neurons (e.g. mediated by CGRP) or that sensory neurons are not involved in the adaptive cytoprotection. It is plausible that these agents may induce NO generation via both sensory neuron-dependent and -independent ways [23]. Nevertheless, reports had indicated contrasting ideas about the contribution of PG formation to the afferent nerve-mediated gastric mucosal protection [24, 25]. In general, the vagal peripheral gastroprotective mechanism is most probably expressed through muscarinic-dependent release of PG and/or NO as well as the efferent function of capsaicin-sensitive afferent fibers releasing CGRP [26]. This also applies to the adaptive cytoprotection by mild irritants [27].

\section{Contemporary Concept on Ischemic Preconditioning, Heat-Shock Proteins and Adaptive Cytoprotection}

When a particular organ is experiencing a brief exposure to ischemia, it would undergo a phenomenon called 'preconditioning. This is the case when the stomach begins to develop increased resistance against severe ischemia/reperfusion or challenge of strong irritants following short and repeated gastric ischemic episodes, which results in a special type of adaptive cytoprotection [28]. A similar restoration of the local mucosal blood flow and increased PG generation as those induced by convention- 
Fig. 1. Mechanism of adaptive cytoprotection induced by mild irritants in the gastric mucosal defense system.

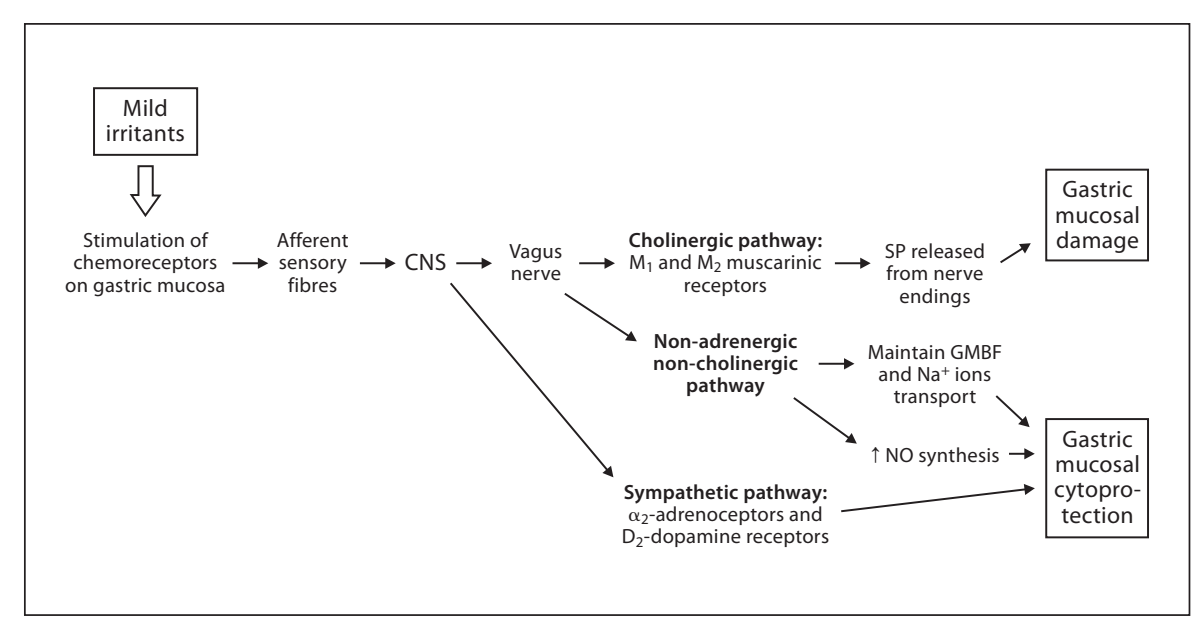

al mild irritants can be observed during such ischemic preconditioning. Likewise, the mediators involved in such gastroprotective activities also include products of the cyclooxygenase and NO synthase enzyme systems. Besides, the gastroprotective and hyperemic actions of standard ischemic preconditioning can be abolished by ablation of the capsaicin-sensitive sensory nerves and restored by exogenous administration of CGRP, implicating participation of the chemosensory system. Hence, ischemic preconditioning now represents a novel and most powerful approach to intervene the mucosal damage caused by severe ischemia/reperfusion and strong irritants in the stomach. In fact, other new ideas such as heat acclimation that could impose constitutive cytoprotection against stressors also indicate a similar concept of adaptive cytoprotection [29]. The potential extrapolation of such new discovery to gastroprotection and further correlation with the chemosensory system may lead to a new avenue in developing effective therapeutic approaches.

Heat-shock proteins (HSP) are crucial for the maintenance of cellular homeostasis during normal cell growth and survival following cellular stresses [30]. Among these, the HSP70 family of proteins is most renowned to exert gastroprotective effects in the gastric mucosa. During the HPA axis activation, the sympathetic signal that has been elicited will cause the relevant stress response in target peripheral tissues, including the gastric mucosa. HSP70 expression in both the vasculature and in mucosal cells will impose a strong resistance against the negative hemodynamic changes and other stress-associated deterioration of the mucosal structures. Both ischemic preconditioning and mild irritant-induced adaptive cyto- protection could trigger the production of HSP, which results in increased tolerance against the insults of strong irritants or stress.

\section{Conclusion}

Gastroprotection and in particular adaptive cytoprotection by mild irritants in the stomach is a classical approach to deal with the mucosal injury caused by stress or strong irritants. From the traditional concept of the PG-based mode of action, diverse mechanisms of the mild irritants have been unveiled throughout the years, including a strong association to the brain-gut axis. Various components of the vagus nerve, sensory afferent neurons and enteric nervous system, including their respective local mediators, have different degrees of contribution to the adaptive cytoprotective processes (summarized in fig. 1). Contemporary concepts of adaptive cytoprotection such as ischemic preconditioning and the correlation with the HSP have also been discussed. It is imperative that future gain in knowledge on adaptive cytoprotection and the brain-gut axis can enable us to establish a better approach to fight against gastric mucosal damages in an effective and safer manner.

\section{Disclosure Statement}

No conflicts of interest to declare. 


\section{References}

1 Robert A, Nezamis JE, Lancaster C, Hanchar AJ: Cytoprotection by prostaglandins in rats. Prevention of gastric necrosis produced by alcohol, $\mathrm{HCl}, \mathrm{NaOH}$, hypertonic $\mathrm{NaCl}$, and thermal injury. Gastroenterology 1979;77: 433-443.

2 Szabo S: Experimental basis for a role for sulfhydryls and dopamine in ulcerogenesis: a primer for cytoprotection-organoprotection. Klin Wochenschr 1986;64(suppl 7):116-122.

3 Chaudhury TK, Robert A: Prevention by mild irritants of gastric necrosis produced in rats by sodium taurocholate. Dig Dis Sci 1980;25:830-836.

4 Konturek SJ, Brzozowski T, Piastucki I, Radecki T, Dembinski A, Dembinska-Kiec A: Role of locally generated prostaglandins in adaptive gastric cytoprotection. Dig Dis Sci 1982;27:967-971.

5 Foschi D, Castoldi L, del Soldato P, Musazzi M, Callioni F, Rovati V, Trabucchi E, Montorsi W: Effects of autonomic nervous system on gastric damage by ethanol in the rat. Dig Dis Sci 1989;34:688-693.

6 Holzer P, Sametz W: Gastric mucosal protection against ulcerogenic factors in the rat mediated by capsaicin-sensitive afferent neurons. Gastroenterology 1986;91:975-981.

7 Holzer P, Guth PH: Neuropeptide control of rat gastric mucosal blood flow: increase by calcitonin gene-related peptide and vasoactive intestinal polypeptide, but not substance $\mathrm{P}$ and neurokinin A. Circ Res 1991;68:100105.

8 Stead RH, Dixon RF, Bramwell NH: Mast cells are closely apposed to nerves in the human gastrointestinal mucosa. Gastroenterology 1989;97:575-585.

9 Whittle BJR, Lopez-Belmonte J, Moncada S: Regulation of gastric mucosal integrity by endogenous nitric oxide: interaction with prostanoids and sensory neuropeptides in the rat. Br J Pharmacol 1990;99:607-611.

10 Esplugues JV, Whittle BJR, Moncada S: Modulation by opioids and by afferent sensory neurons of prostanoid protection of the rat gastric mucosa. Br J Pharmacol 1992;106: 846-852.
11 Cho CH, Ogle CW: The effect of zinc sulphate on vagally-induced mast cell changes and ulcers in the rat stomach. Eur J Pharmacol 1976;43:315-322.

12 Henagan JM, Smith GS, Seidel ER, Miller TA: Influence of vagotomy on mucosal protection against alcohol-induced gastric damage in the rat. Gastroenterology 1984;87: 903-908.

13 Cho CH, Chen BW, Hui WM, Lam SK, Ogle $\mathrm{CW}$ : The role of the vagus nerve in the protective action of acid inhibitors on ethanolinduced gastric mucosal damage in rats. J Gastroenterol Hepatol 1992;7:178-183.

14 Okumura T, Uehara A, Okumura K, Namiki M: Site-specific formation of gastric ulcers by the electric stimulation of the left or right gastric branch of the vagus nerve in the rat. Scand J Gastroenterol 1990;25:834-840.

15 Ko JKS, Cho CH, Ogle CW: The vagus nerve and its non-cholinergic mechanism in the modulation of ethanol-induced gastric mucosal damage in rats. J Pharm Pharmacol 1994;46:29-32.

16 Sikiric P, Rotkvic I, Mise S, Krizanac S, Suchanek E, Petek M, Gjuris V, Geber J, Tucan-Foretic M, Udovicic I, Anic T, Balen I: The influence of dopamine agonists and antagonists on gastric lesions in mice. Eur J Pharmacol 1987;144:237-239.

17 Ko JKS, Cho CH: The mechanistic pathway of gastric adaptive cytoprotection: a study on different components of the autonomic nervous system. J Auton Pharmacol 1995;15: 205-214.

18 Bhandare PN, Rataboli V, d'Souza RSD: Dual action of clonidine on ethanol-induced gastric lesions: is the imidazoline-preferring receptor involved? Eur J Pharmacol 1991; 199:243-245.

19 Yonei Y, Holzer P, Guth PH: Laparotomy-induced gastric protection against ethanol injury is mediated by capsaicin-sensitive sensory neurons. Gastroenterology 1990;99: 3-9.

20 Ottaway CA: Role of the neuroendocrine system in cytokine pathways in inflammatory bowel disease. Aliment Pharmacol Ther 1996;10(suppl 2):10-15.

21 Filaretova LP, Podvigina TT, Bobryshev PY, Bagaeva TR, Tanaka A, Takeuchi K: Hypothalamic-pituitary-adrenocortical axis: the hidden gold in gastric mucosal homeostasis. Inflammopharmacology 2006;14:207-213.
22 Evagelista S, Maggi CA, Meli A: Lack of influence of capsaicin-sensitive sensory fibers on adaptive cytoprotection in rat stomach. Dig Dis Sci 1988;33:1050-1051.

23 Hatakeyama Y, Tomoi M, Ohtsuka M, Shimomura K: Implication of sensory neurons in the diverse mechanisms of adaptive cytoprotection in the rat stomach. Jpn J Pharmacol 1996;70:347-350.

24 Uchida M, Yano S, Watanabe K: The role of capsaicin-sensitive afferent nerves in protective effect of capsaicin against absolute ethanol-induced gastric lesions in rats. Jpn J Pharmacol 1991;55:279-282.

25 Holzer P, Pabst MA, Lippe ITH, Peskar BM, Peskar BA, Livingston EH, Guth PH: Afferent nerve-mediated protection against deep mucosal damage in the rat stomach. Gastroenterology 1990;98:838-848.

26 Taché Y, Yoneda M, Kato K, Király A, Sütö G, Kaneko $\mathrm{H}$ : Intracisternal thyrotropin-releasing hormone-induced vagally mediated gastric protection against ethanol lesions: central and peripheral mechanisms. J Gastroenterol Hepatol 1994;9(suppl 1):S29-S35.

27 Ohno BK, Saeki T, Hayashi H, Hayashi I, Katori M, Murata T, Narumiya S, Saigenji K, Majima M: Adaptive cytoprotection mediated by prostaglandin $I_{2}$ is attributable to sensitization of CRGP-containing sensory nerves. Gastroenterology 2001;120:134-143.

28 Pajdo R, Brzozowski T, Konturek PC, Kwiecien S, Konturek SJ, Sliwowski Z, Pawlik M, Ptak A, Drozdowicz D, Hahn EG: Ischemic preconditioning, the most effective gastroprotective intervention: involvement of prostaglandins, nitric oxide, adenosine and sensory nerves. Eur J Pharmacol 2001; 427:263-276.

29 Horowitz M, Assadi H: Heat acclimationmediated cross-tolerance in cardioprotection: do HSP70 and HIF-1 $\alpha$ play a role? Ann NY Acad Sci 2010;1188:199-206.

30 Rokutan K, Hirakawa T, Teshima S, Nakano Y, Miyoshi M, Kawai T, Konda E, Morinaga $\mathrm{H}$, Nikawa T, Kishi K: Implications of heat shock/stress proteins for medicine and disease. J Med Invest 1998;44:137-147. 